\title{
Mechanically-induced transport switching effect in graphene-based nanojunctions
}

\author{
T. Kawai, ${ }^{1}$ M. Poetschke, ${ }^{2}$ Y. Miyamoto, ${ }^{3}$ C. G. Rocha, ${ }^{2}$ S. Roche, $, 4,4$ and G. Cuniberti ${ }^{2,6}$ \\ ${ }^{1}$ Green Innovation Research Laboratories, NEC Corporation, 34 Miyukigaoka, Tsukuba, Ibaraki 305-8501, Japan \\ ${ }^{2}$ Institute for Materials Science and Max Bergmann Center of Biomaterials, Dresden University of Technology, 01062 Dresden, Germany \\ ${ }^{3}$ Nanosystem Research Institute, National Institute Advanced Industrial Science and Technology (AIST) Central 2, 1-1-1 Umezono, Tsukuba, \\ 305-8568 Japan and Graphene Division, Technology Research Association for Single Wall Carbon Nanotube (TASC) Central 2, \\ 1-1-1 Umezono, Tsukuba, 305-8568, Japan \\ ${ }^{4}$ CIN2 (ICN-CSIC) and Universitat Autónoma de Barcelona, Catalan Institute of Nanotechnology, Campus de la UAB - 08193 \\ Bellaterra (Barcelona), Spain \\ ${ }^{5}$ ICREA, Institució Catalana de Recerca i Estudis Avancats - 08010 Barcelona, Spain \\ ${ }^{6}$ Division of IT Convergence Engineering, POSTECH, Pohang 790-784, Republic of Korea
}

(Received 11 May 2011; published 23 June 2011)

\begin{abstract}
We report a theoretical study suggesting a novel type of electronic switching effect, driven by the geometrical reconstruction of nanoscale graphene-based junctions. We considered junction structures that have alternative metastable configurations transformed by rotations of local carbon dimers. The use of external mechanical strain allows a control of the energy-barrier heights of the potential profiles and also changes the reaction character from endothermic to exothermic or vice versa. The reshaping of the atomic details of the junction encode binary electronic "on" or "off" states, with on/off transmission ratio that can reach up to $10^{4}-10^{5}$. Our results suggest the possibility to design modern logical switching devices or mechanophore sensors, monitored by mechanical strain and structural rearrangements.
\end{abstract}

DOI: 10.1103/PhysRevB.83.241405

PACS number(s): 81.05.ue, 62.25.-g, 72.80.Vp

Current strategies for miniaturization of logic and memory devices envision the ultimate limit that is the atomic-scale control. Several experimental groups have demonstrated the capability of controlling the current flow through single molecular junctions, ${ }^{1-5}$ highlighting promising applications in realms such as nanotechnology and medicine. Outstanding switching properties have been recently revealed in atomic-scale metallic contacts disposed in a three-terminal architecture where the conductance was mechanically switched between "on" and "off" states at room temperature. ${ }^{6}$ Other attractive materials such as photochromic organic molecules have been widely used for designing LCD displays and liquid crystals since their transport features can be driven by light irradiation ${ }^{7}$ or current pulses. $^{8}$ In the quest of designing efficient atomicscale switching devices, graphene-based materials deserve special attention due to a peculiar one-atom-thick planar geometry combined with exceptional electronic, ${ }^{9}$ mechanical, and thermal properties. ${ }^{10-12}$ As a result, a wealth of novel phenomena is steadily unveiled in different fields, including spintronics, ${ }^{13}$ ac transport, ${ }^{14-16}$ or thermoelectrics. ${ }^{17}$

However, the use of graphene for designing logical switching applications is severely limited by the absence of energy gap. Using advanced lithographic techniques ${ }^{18,19}$ or chemically-assisted bottom-up fabrication strategies, ${ }^{20}$ the fabrication of graphene nanoribbons (GNRs) combined with the possibility of band-gap engineering their electronic structure have opened a promising route towards the design of more efficient switching devices. ${ }^{21}$ Patterned monolayer graphene nanoconstriction-based field-effect transistors were successfully synthesized, confirming that structural confinement can induce energy gap opening and a sharp increase in the on/off ratio $\left(\sim 10^{4}\right){ }^{22}$ GNRs-based electromechanical and electromagnetic switches were also designed, evidencing the possibility of switching principles of different physical natures. ${ }^{23,24}$ Recently, an efficient and reversible current-induced switching mechanism was reported in large area-graphene-based devices, allowing for the design of nonvolatile memory elements. ${ }^{25}$ The origin of this switching phenomenon was tentatively related to the formation and breaking of carbon atomic chains bridging two sections of the sample. Additional control parameters can be considered to modulate in a reversible fashion the binary switching properties of molecular junctions. For instance, recent studies have confirmed that mechanical strain can effectively monitor the electronic structure and transport response of carbon-based materials. ${ }^{26-30}$ In particular, for graphene that are gapless materials, the use of mechanical forces as control parameters can establish important strategies for envisioning band gap engineering in graphene structures.

Despite efforts to develop monitored switching devices, the understanding of intrinsic operating mechanisms and their relation with the atomic-scale features are open issues requiring advanced theoretical investigation. In particular, there is a need to investigate the deep connection between reversible modifications occurring at the atomic scale and the resulting electronic behavior of devices exposed to external perturbations. ${ }^{31-34}$ In this paper, the inherent switching mechanism of physical reactions involving graphene-etched junctions is dynamically probed using theoretical simulations. Our goal is to identify optimum physical conditions for tuning the conductance between binary switching states. A physical reaction is simulated by examining the potential profile on the coordination axis of the rotating carbon dimers under applied mechanical stress needed for improving the switching features of the device. The associated activation energy for the reaction and its ground state are determined by the derivation of minimum energy paths (MEP). Mechanical strain is subsequently used to modulate the energy onsets and barrier heights that characterize the structural transition. Regarding the transport response of the system, we establish clear thresholds between on and off states as we monitor 


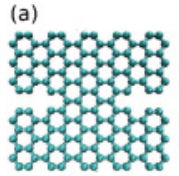

(b)

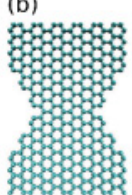

$\mathrm{p}=0.0$

FIG. 1. (Color online) The corresponding (ball-and-stick) atomic configurations for initial $(p=0)$, intermediate $(p=0.5)$, and final states ( $p=1.0)$ for (a) bi- and (b) tri-switching structures. Panel (c): Ball-and-stick model of graphene-based junctions under uniaxial mechanical forces (arrows).

their electronic transmission along the whole reaction path. Prominent switching behavior can be achieved depending on the atomic widths of the nanostructures and amount of applied strain. Our findings ensure a promising way for designing ultimate molecular switching devices based on strained graphene. We also extend graphene's applicability to another scientific frontier, the production of mechanophore sensors based on etched graphene samples. Such special materials consist of systems where their reaction path depends on the strength and nature of the applied force. ${ }^{35}$

Minimum-energy-path results. In what follows, we performed systematic analysis of transport properties and mechanical activity of two different graphene constrictions, where carbon dimers bridge the gap between two semi-infinite graphene ribbons. Here, we refer to the constriction with two (three) $\mathrm{C}-\mathrm{C}$ bonds linking the graphene segments as a bi- (tri-) switch configuration. A minimum energy path that resolves the system undergoing a structural rearrangement of atoms can be tracked assuming two metastable configurations: a graphene nanojunction made by pure hexagons (initial state) and a defective constriction composed of pentagon-heptagon pairs (final state). The variable $p$ is associated to the normalized reaction coordinate where $p=0$ corresponds to the initial anchor state and $p=1.0$ defines the ending of the reaction. Ball-and-stick schematic pictures for initial, intermediate, and final states are displayed in Figs. 1(a) and 1(b). The physical reaction consists of rotating the $\mathrm{C}-\mathrm{C}$ bonds located on the "neck" of the initial hexagonal junction by $90^{\circ}$, generating rings of Stone-Wales defects in the final configuration. After the rotation, we observe that the optimal lattice constant along the axial direction suffers a small expansion of $\sim 0.7 \AA$, characterizing an endothermic-like reaction. The transition route linking these two configurations states can be located using the nudged elastic band (NEB) method. ${ }^{36}$ The method gathers the MEP by constructing a set of replicas of the system between the initial and final states. A spring interaction between consecutive images is included to ensure the continuity of the path. Optimization procedures that minimize the forces acting on the images are implemented to obtain the MEP.

Our results obtained for the two switching systems are shown in Fig. 2. The total energy variation along the whole

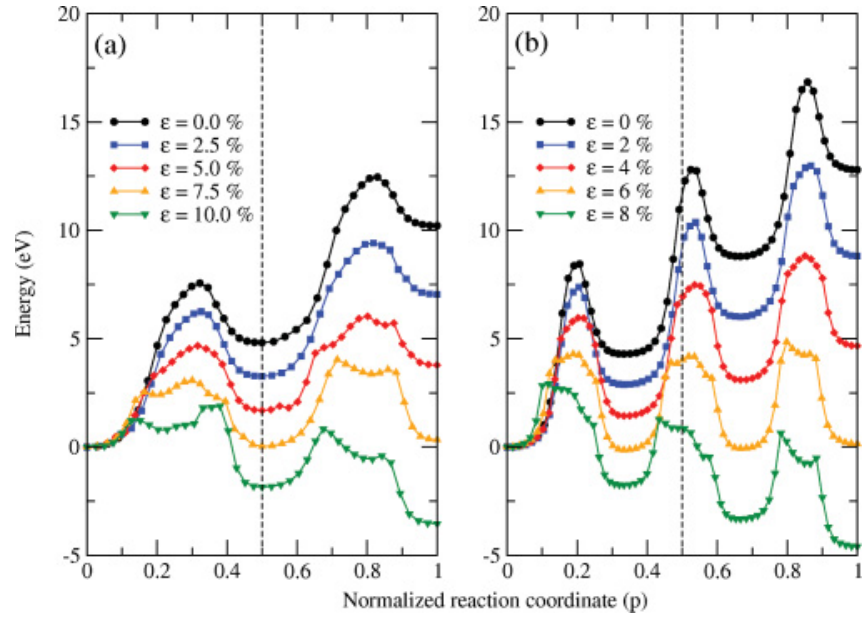

FIG. 2. (Color online) Minimum energy paths calculated for (a) bi- and (b) tri- switching structures for different values of mechanical strain. The vertical dotted lines in the graphs denote the corresponding positions of intermediate states $(p=0.5)$ in the reaction coordinate. Reference energy for the initial configuration was set to $0 \mathrm{eV}$.

trajectory is mapped as a function of the reaction coordinate $(p)$ and the reference energy for the initial state is set to $0 \mathrm{eV}$. The structures are also submitted to uniaxial mechanical forces under DFTB frame, ${ }^{37}$ and we demonstrate the ability of controlling the MEP profiles by applying such perturbation. A schematic representation of the applied forces is shown in Fig. 1(c). We applied mechanical strain by pulling away the atoms located at upper and lower edges at incremental values and further fixing them. The atomic geometries, except for the edge-constrained atoms, are subsequently relaxed under the conditions of the NEB method. The equivalent mechanical energy resulting from the stretching is defined as $E_{M}=$ $-\Delta \vec{L} \cdot \vec{F}$, being $\vec{F}$ the external force and $\Delta L=L-L_{0}$, with $L$ and $L_{0}$ the deformed and initial equilibrium lattice constant parameters along the axial direction, respectively. The strain is calculated as $\epsilon=\Delta L / L_{0}$.

For unstrained systems, the ground state for both switching systems is the initial hexagonal junction. For bi-switching configuration, two humps can be observed along the reaction path, while three others appear for tri-switching arrangement. Each one of those humps is associated to the amount of energy required to rotate one $\mathrm{C}-\mathrm{C}$ bond by $90^{\circ}$ in the junction. After the rotation, we observe that the optimal lattice constant along the axial direction suffers a small expansion of $\sim 3 \%$, characterizing an endothermic reaction. The energy barrier heights for the complete transition are estimated as $12.4 \mathrm{eV}$ and $16.8 \mathrm{eV}$ for bi-switching and tri-switching structures, respectively. The barrier heights, as well as the energies of initial/final configurations, can be strongly modulated with the aid of mechanical strain. From the pictures, we can see that the barrier heights are considerably reduced as stretching takes place. At tensile strains of $7.5 \%$ (6\%) for bi- (tri-) switching structure, the barrier heights for both structures decrease from $12.4 \mathrm{eV}(16.8 \mathrm{eV})$ to $4.0 \mathrm{eV}(4.8 \mathrm{eV})$, and the anchor states are set almost evenly in energy. As the mechanical strain continues to increase, the ground state is switched from the hexagonal junction to the 5-7 defective configuration and the reaction 


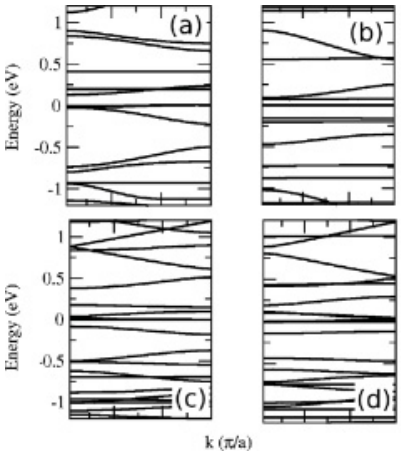

(e)

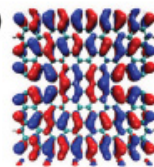

(g)

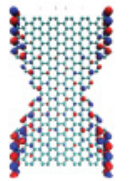

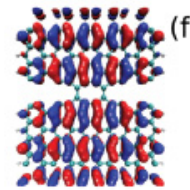

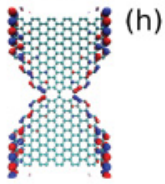

FIG. 3. (Color online) Band structures obtained for initial [(a) and (c)] and final [(b) and (d)] states of a bi- (upper panels) and tri- (lower panels) switching graphene junctions under strain (7.5\% and 6\%, respectively). Electronic wave functions calculated at $\Gamma$ point at the energies are shown in (e) $-0.74 \mathrm{eV}$, (f) $-0.72 \mathrm{eV}$, (g) $-0.02 \mathrm{eV}$, and (h) $-0.01 \mathrm{eV}$. Red (dark gray) and light blue (light gray) colors correspond to positive and negative coefficients, respectively.

becomes exothermic-like. The formation of 5-7 defect lines reduces the number of the bonds connecting the two graphene segments and this dwindles the energy loss in response to the elongation.

Up to this point, we evidenced that the physical reactions involving graphene junctions under strains of 6\%-7.5\% are energetically favorable to be triggered since the total energies of initial and final states are comparable and the barrier heights are considerably reduced. We now explore if such strained systems reveal optimum switching behavior through electronic structure analysis. This issue is investigated by following how the electronic structure and transport responses change along the reaction coordinate path. The efficiency of standard logical switches can usually be measured through the magnitude of on/off transmission rates, controlled by gate voltages. In our case, the reaction coordinate that resolves the dynamic trajectory between the anchor states acts as an additional switching parameter. In this sense, prominent switching responses can also be dynamically tuned as the system evolves along the MEP. An efficient dynamic switch is characterized by finding pronounced differences between the transmission values taken from the initial and final configurations. To represent the electronic structure of both extreme states, we adopted self-consistent-charge density functional-based tight binding theory (DFTB $)^{38,39}$ and the conductance was subsequently calculated using Landauer formula written in terms of Green's functions. ${ }^{40}$

Figure 3 depicts the energy relation dispersions calculated for initial [Figs. 3(a) and 3(c)] and final states [Figs. 3(b) and 3(d)] for bi-switching (upper panels) and tri-switching (lower panels) systems. The Fermi energy is set at $0 \mathrm{eV}$. The atomic arrangement along the edges of both junctions follows a zigzag shape, and hydrogen atoms are used to saturate dangling bonds situated outside the constriction domain. The nonsaturated dangling bonds give rise to flat bands at the Fermi level. This can be confirmed through the electronicwave function plots displayed on the lower panels [Figs. 3(g) and 3(h)] for the tri-switching example. The wave functions are calculated at the $\Gamma$ point for energies around $E_{F}=0.0 \mathrm{eV}$. In fact, the electronic distribution is highly concentrated on the edges. Such bands are rather robust and do not respond
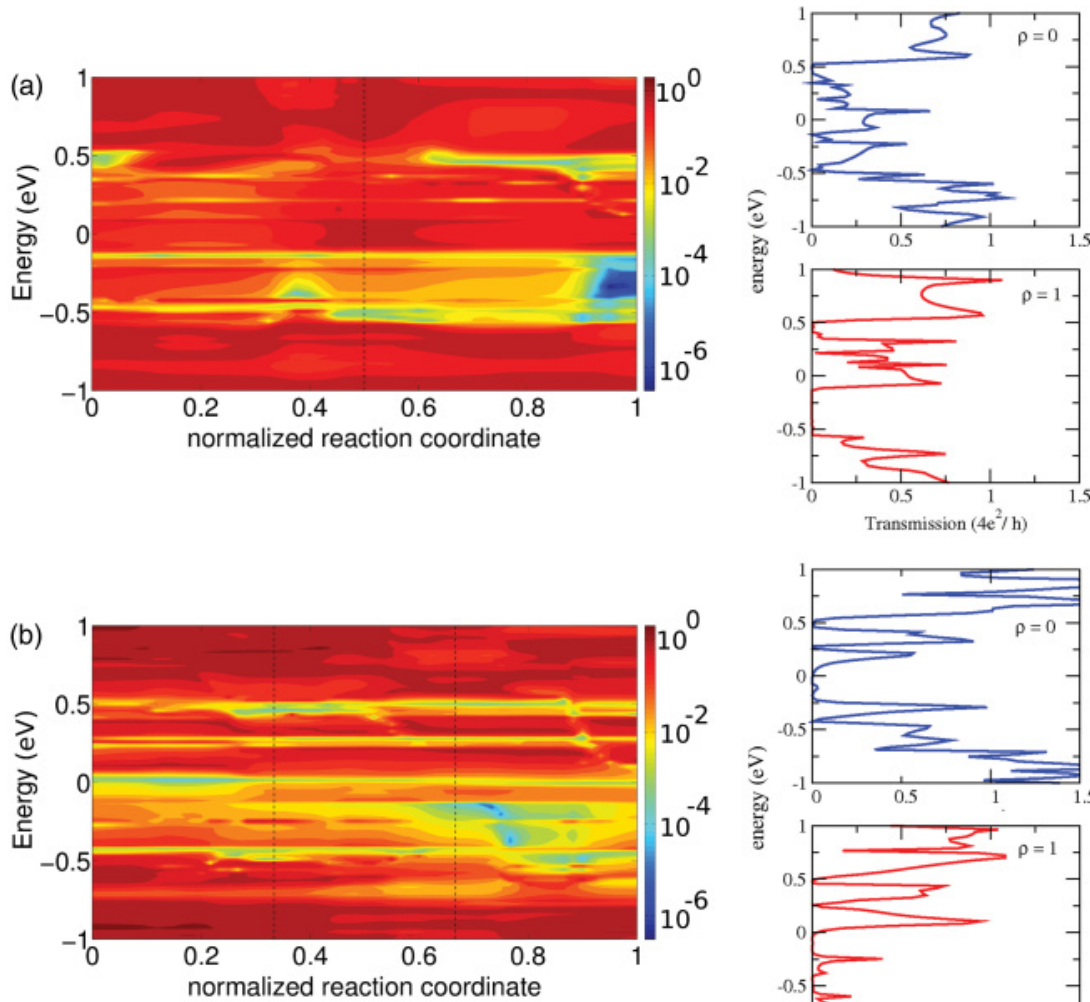

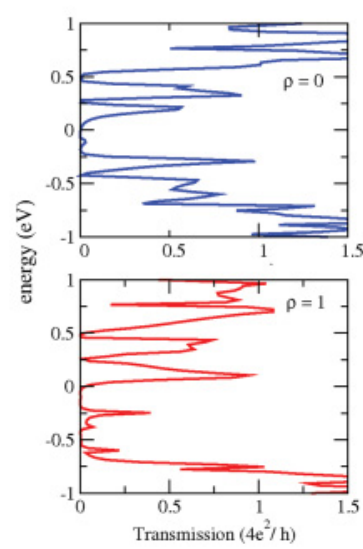

$241405-3$
FIG. 4. (Color online) Decadic logarithm of the transmission as a function of reaction coordinate and Fermi energy for a (a) bi- and (b) tri-switching structure under mechanical strain $(7.5 \%$ and $6.0 \%$, respectively). Panels beside the contour plots display the transmission curves as a function of energy at initial $(\rho=0)$ and final $(\rho=1)$ reaction coordinates for (upper panels) bi- and (bottom panels) tri-switching configurations. 
to the structural modification of rotating carbon dimers. This indicates that favorable switching features cannot be achieved at $E_{F}=0.0 \mathrm{eV}$ for both nanojunctions.

For the bi-switching arrangement, outstanding switching properties can be highlighted in the energy range of $-0.5<$ $E<-0.1 \mathrm{eV}$. The sub-band dispersion within this range shown in Fig. 3(a) is considerably reduced as the bonds rotate, characterizing a potential transition between on [Fig. 3(a)] and off [Fig. 3(b)] states. To rationalize these findings, the electronic wave functions calculated at the $\Gamma$ point are shown for particular energies in the bi-switching example [Figs. 3(e) and 3(f), lower panels]. We observe a highly delocalized spatial distribution of conduction channels for the initial frame, whereas two disconnected orbital patterns are obtained at the ending of the reaction. Rotating the horizontal $\mathrm{C}-\mathrm{C}$ bonds of the hexagonal bi-switching junction disrupts the superposition of the molecular orbitals on the constriction. The system behaves, then, as two isolated semi-infinite graphene sections and the electronic channel is blocked. On the other hand, the electronic structure of tri-switching arrangement seems to exhibit weaker switching behavior. Comparing the energy relations displayed in Figs. 3(c) and 3(d), one can see a significant resemblance within the whole spectrum, which means that the structural reaction does not cause significant impact on the electronic response of such junctions.

The precise evaluation of the on/off transmission rates is obtained through Fig. 4, which shows the conductance contour plots as a function of the Fermi energy and reaction coordinates obtained for both strained samples. The results reveal once more the excellent binary switching behavior of bi-switching structure in contrast to tri-switching case. The gradient color located on the energy range of $-0.5<E<$ $-0.1 \mathrm{eV}$ unveils a gradual exchange between on (red color online) and off (blue color online) states with respect to the reaction coordinate path. The maximum transmission rate is determined as $10^{4}-10^{5}$. Assuming that the final state can be stabilized, the transition between high and low transmission states can also be induced through the use of some external gate voltages. Differently, tri-switching configuration manifests a robust transport response with minor changes appearing in the same energy range. Lower transmission values are evidenced only for a few intermediate reaction frames, indicating that the weaker switching capability of such a system can be better manipulated via gate voltages.

Conclusion. We reported the manifestation of binary switching behavior on graphene nanojunctions that experience a physical reaction capable of rearranging locally the atomic structure of the systems. The junctions are also exposed to uniaxial mechanical stretching, which tunes the physical characteristics of the reaction such as energy barrier heights and ground state. As the reaction occurs, we observe that narrower junctions reveal promising on/off transmission rates, reaching values up to $10^{4}-10^{5}$, which is in agreement with current experimental measurements performed in graphene nanoconstrictions. ${ }^{22}$ Our results also support the interpretation of recent experiments carried on atomic quantum transistors where self-stabilizing contact reconstruction can be mechanically driven. ${ }^{6}$

Acknowledgments. This work was partially funded by the Alexander von Humboldt Foundation, the European Union, the Free State of Saxony (SAB Project No. A2-13996/2379), the NANOSIM-GRAPHENE (Project No. ANR-09-NANO-01601), and the South Korean Ministry of Education, Science, and Technology Program (WCU ITCE Project No. R31-2008000-10100-0).
${ }^{1}$ J. Chen et al., Science 286, 1550 (1999).

${ }^{2}$ E. Scheer et al., Nature 394, 154 (1998).

${ }^{3}$ F.-Q. Xie et al., Phys. Chem. 233, 153 (2006).

${ }^{4}$ D. Nozaki and G. Cuniberti, Nano Res. 2, 648 (2009).

${ }^{5}$ F. Patolsky et al., Proc. Natl. Acad. Sci. U.S.A. 101, 14017 (2004).

${ }^{6}$ F.-Q. Xie et al., Nano Lett. 8, 4493 (2008).

${ }^{7}$ T. Brakemann et al., J. Biol. Chem. 285, 14603 (2010).

${ }^{8}$ C. Zhang et al., Phys. Rev. Lett. 92, 158301 (2004).

${ }^{9}$ Y. W. Tan et al., Phys. Rev. Lett. 99, 246803 (2007); A. Lherbier et al., ibid. 100, 036803 (2008).

${ }^{10}$ K. S. Novoselov et al., Science 306, 666 (2004).

${ }^{11}$ A. H. Castro Neto et al., Rev. Mod. Phys. 81, 109 (2009); M. Lemme, Sol. Stat. Phenom. 156-158, 499 (2010).

${ }^{12}$ J. Zimmermann et al., Phys. Rev. B 78, 045410 (2008).

${ }^{13}$ Y.-W. Son et al., Nature (London) 444, 347 (2006); S. Lakshmi et al., Phys. Rev. B 80, 193404 (2009).

${ }^{14}$ C. G. Rocha et al., Phys. Rev. B 81, 115435 (2010).

${ }^{15}$ E. Prada et al., Phys. Rev. B 80, 245414 (2009).

${ }^{16}$ R. Zhu, and H. Chen, Appl. Phys. Lett. 95, 122111 (2009).

${ }^{17}$ Y. M. Zuev et al., Phys. Rev. Lett. 102, 096807 (2009).

${ }^{18}$ S. S. Datta et al., Nano Lett. 8, 1912 (2008).

${ }^{19}$ M. C. Lemme et al., ACS Nano 3, 2674 (2009).

${ }^{20}$ T. Shimizu, J. Haruyama, D. C. Marcano, D. V. Kosinkin, J. M. Tour, K. Hirose, and K. Suenaga, Nature Nanotech. 6, 45 (2011); S. Roche, ibid. 6, 8 (2011).
${ }^{21}$ A. Cresti, N. Nemec, B. Biel, G. Niebler, F. Triozon, G. Cuniberti, and S. Roche, Nano Research 1, 361 (2008).

${ }^{22}$ Y. Lu et al., Small 6, 2748 (2010).

${ }^{23}$ K. M. Milaninia et al., Appl. Phys. Lett. 95, 183105 (2009).

${ }^{24}$ M. Dragoman et al., J. Appl. Phys. 105, 054309 (2009).

${ }^{25}$ B. Standley et al., Nano Lett. 8, 3345 (2008).

${ }^{26}$ S. Y. Quek et al., Nat. Nanotech. 4, 230 (2009).

${ }^{27}$ Z. H. Ni et al., ACS Nano 3, 483 (2009).

${ }^{28}$ M. Poetschke et al., Phys. Rev. B 81, 193404 (2010).

${ }^{29}$ T. M. G. Mohiuddin, et al., Phys. Rev. B 79, 205433 (2009).

${ }^{30}$ V. M. Pereira and A. H. Castro Neto, Phys. Rev. Lett. 103, 046801 (2009); V. M. Pereira, N. M. R. Peres, and A. H. Castro Neto, Phys. Rev. B 80, 045401 (2009).

${ }^{31}$ L. A. Agapito and H.-P. Cheng, J. Phys. Chem. C 111, 14266 (2007).

${ }^{32}$ C. Yin et al., J. Chem. Phys. 131, 234706 (2009).

${ }^{33}$ A. Hashimoto et al., Nature (London) 430, 870 (2004).

${ }^{34}$ B. I. Yakobson et al., Carbon 38, 1675 (2000).

${ }^{35}$ J. R. Arino et al., J. Am. Chem. Soc. 132, 10609 (2010).

${ }^{36}$ G. Henkelman et al., J. Chem. Phys. 113, 9901 (2000).

${ }^{37}$ C. H. Xu et al., J. Phys. Condens. Matter 4, 6047 (1992).

${ }^{38}$ M. Elstner et al., Phys. Rev. B 58, 7260 (1998).

${ }^{39}$ G. Seifert et al., Int. J. Quantum Chem. 58, 185 (1996).

${ }^{40}$ M. Buongiorno Nardelli, Phys. Rev. B 60, 7828 (1999). 